\title{
ON THE DUALS OF LEBESGUE-BOCHNER $L^{p}$ SPACES
}

\author{
BAHAETTIN CENGIZ
}

(Communicated by Palle E. T. Jorgensen)

\begin{abstract}
Let $(X, \mathscr{A}, \mu)$ be an arbitrary positive measure space. We prove that there exist an extremally disconnected (locally) compact Hausdorff space $Y$ and a perfect (regular) Borel measure $\nu$ on $Y$ such that $L^{p}(\mu, E) \simeq L^{p}(\nu, E)$ for all $1 \leq p<\infty$ and any Banach space $E$. If $E^{*}$ is separable, then $L^{p}(\mu, E)^{*} \simeq L^{q}\left(\mu, E^{*}\right)$ for all $1<p<\infty, \frac{1}{p}+\frac{1}{q}=1$, and $L^{1}(\mu, E)^{*} \simeq$ $L^{\infty}\left(\nu, E^{*}\right) \simeq C\left(\beta Y, E_{*}^{*}\right)$, where $E_{*}^{*}$ denotes $E^{*}$ endowed with the weak * topology. In particular $L^{1}(\mu)^{*} \simeq L^{\infty}(\nu)$.
\end{abstract}

Let $(X, \mathscr{A}, \mu)$ be an arbitrary measure space ${ }^{1}$ and $E$ a Banach space. We shall denote the Lebesgue-Bochner spaces $L^{p}(X, \mathscr{A}, \mu, E), 1 \leq p \leq \infty$, by $L^{p}(\mu, E)$, and by $L^{p}(\mu)$ when $E$ is the scalar field, if there is no chance of confusing the underlying measurable spaces. For the definitions and properties of these spaces we refer to [2]. We shall write $E \simeq F$ to mean that the Banach spaces $E$ and $F$ are isometric. $E^{*}$ will denote the dual of $E$.

Let $1 \leq p<\infty$ and $q$ be such that $\frac{1}{p}+\frac{1}{q}=1$. For $g \in L^{q}\left(\mu, E^{*}\right)$ we define $\Phi_{g}$ on $L^{p}(\mu, E)$ by the equation $\Phi_{g}(f)=\int_{X}\langle f, g\rangle d \mu, f \in L^{p}(\mu, E)$.

In the case of scalar-valued functions, it is common knowledge that the mapping $g \rightarrow \Phi_{g}$ is a linear isometry from $L^{q}(\mu)$ into the dual space $L^{p}(\mu)^{*}$. It is surjective for all $1<p<\infty[4$, p. 286]. For $p=1$, examples show that, in general, this mapping need not be surjective (e.g. [5, p. 349]); that is, there may not be enough functions in $L^{\infty}(\mu)$ to represent all the elements of $L^{1}(\mu)^{*}$. However, if the measure space is decomposable (meaning that $X$ is the disjoint union of measurable subsets, $X=\bigcup_{i \in I} X_{i}$, with $\mu\left(X_{i}\right)<\infty$ for all $i$, and $\mu(A)=\sum_{i \in I} \mu\left(A \cap X_{i}\right)$ for every measurable set $A$ of finite measure), then the mapping under consideration is surjective $[5$, p. 353] (or $[4$, p. 290]). Thus, for some nondecomposable measure spaces, $L^{1}(\mu)^{*}$ cannot be represented as $L^{\infty}(\mu)$ (via integral). However, J. Schwartz [7] has found a representation of it as a space of certain scalar countably additive set functions defined on the family of measurable sets of finite measure.

In the case of vector-valued functions, $L^{p}(\mu, E)^{*} \simeq L^{q}\left(\mu, E^{*}\right)$ for all $1 \leq$ $p<\infty$, if either $(X, \mathscr{A}, \mu)$ is decomposable and $E^{*}$ is separable [3, p. 282] or

Received by the editors October 22, 1989.

1980 Mathematics Subject Classification (1985 Revision). Primary 46E30, 28B05; Secondary $46 \mathrm{E} 40$.

${ }^{1}$ In this article all measures are assumed to be positive. 
$(X, \mathscr{A}, \mu)$ is $\sigma$-finite and $E^{*}$ has the Radon-Nikodym property with respect to $\mu[2$, p. 98$]$.

Following [1], we shall call a Borel measure $\nu$ on an extremally disconnected locally compact Hausdorff space $Y$, perfect if

(i) every nonempty clopen (closed and open) set has positive measure,

(ii) every nowhere dense Borel set has measure zero, and

(iii) every nonempty clopen set contains another clopen set with finite measure.

In this article we shall replace a given arbitrary measure space $(X, \mathscr{A}, \mu)$ by a new measure space $(Y, \mathscr{B}, \nu)$ which will not affect the spaces $L^{p}(\mu, E)$; that is, $L^{p}(\mu, E) \simeq L^{p}(\nu, E)$ for all $1 \leq p<\infty$, and will "enlarge" $L^{\infty}(\mu, E)$ so that at least in the scalar case, $L^{\infty}(\nu)$ will have enough functions to represent all the elements of $L^{1}(\mu)^{*}$. Moreover, $Y$ will be an extremally disconnected (locally) compact Hausdorff space, $\mathscr{B}$ will be the Borel algebra of $Y$, and $\nu$ will be a perfect (regular) Borel measure. Actually, we will have more: if $E^{*}$ is separable then $L^{p}(\mu, E)^{*} \simeq L^{q}\left(\mu, E^{*}\right)$ for $1<p<\infty, \frac{1}{p}+\frac{1}{q}=1$, and $L^{1}(\mu, E)^{*} \simeq L^{\infty}\left(\nu, E^{*}\right)$.

Aside from the above-mentioned goal, we shall also obtain a generalization of a theorem by Cambern and Greim [1] which states that for any extremally disconnected compact Hausdorff space $Z$, any perfect measure $\lambda$ on the Borel algebra of $Z$, and any Banach space $E, L^{1}(\lambda, E)^{*} \simeq C\left(Z, E_{*}^{*}\right)$ where $E_{*}^{*}$ denotes $E^{*}$ endowed with the weak ${ }^{*}$ topology, and $C\left(Z, E_{*}^{*}\right)$ denotes the space of all continuous functions from $Z$ into $E_{*}^{*}$ provided with the supremum norm.

Let $(X, \mathscr{A}, \mu)$ be a finite measure space, $\pi$ the quotient mapping from $\mathscr{A}$ onto the measure algebra $\mathscr{A} / \mu$, and $\delta$ the Stone representation of $\mathscr{A} / \mu$ onto the algebra of all clopen subsets of the Stonean space $\Omega$ of $\mathscr{A} / \mu$. For $A \in \mathscr{A}$ define $\hat{\mu}(\widehat{A})=\mu(A)$ where $\widehat{A}=\delta(\pi(A))$. The space $\Omega$ is extremally disconnected; $\hat{\mu}$ is a measure on the Boolean algebra of all clopen subsets of $\Omega$ and extends uniquely to a perfect regular Borel measure on $\Omega$ that we shall also denote by $\hat{\mu}[6$, p. 120]. As can be checked very easily $\hat{\mu}(\mathrm{Cl} U)=\hat{\mu}(U)$ for every open set, where $\mathrm{Cl} U$ denotes the closure of $U$. It is also easy to see that for every Borel set $B$ there exists a clopen set $C$ such that the symmetric difference $B \Delta C$ has measure zero. Let $\mathscr{B}$ be the Borel algebra of $\Omega$. In the sequel we shall refer to the measure space $(\Omega, \mathscr{B}, \hat{\mu})$ as the perfect measure space associated with the finite measure space $(X, \mathscr{A}, \mu)$.

Clearly, for each $1 \leq p<\infty$ and each Banach space $E$, the mapping

$$
\sum_{i=1}^{n} x_{i} \chi_{A_{i}} \rightarrow \sum_{i=1}^{n} x_{i} \chi_{\widehat{A}_{i}}, \quad x_{i} \in E, A_{i} \in \mathscr{A},
$$

is a linear isometry between dense subspaces of $L^{p}(\mu, E)$ and $L^{p}(\hat{\mu}, E)$ and hence they are isometric. The same mapping can also be used to establish an isometry between $L^{\infty}(\mu, E)$ and $L^{\infty}(\hat{\mu}, E)$.

Now let $(X, \mathscr{A}, \mu)$ be an arbitrary measure space. We shall call two measurable sets $\mu$-disjoint if their intersection has measure zero. An application of Zorn's lemma can be used to show that there exists a maximal family of mutually $\mu$-disjoint measurable sets with strictly positive finite measure. Any such family will be called a $\mu$-decomposition of the measure space $(X, \mathscr{A}, \mu)$. 
Theorem. Let $(X, \mathscr{A}, \mu)$ be an arbitrary measure space. Then there exist an extremally disconnected locally compact space $Y$ and a perfect (regular) Borel measure $\nu$ on $Y$ such that for any Banach space $E, L^{p}(\mu, E) \simeq L^{p}(\nu, E)$ for all $1 \leq p<\infty$.

Proof. Let $\left\{X_{i}: i \in I\right\}$ be a $\mu$-decomposition of $(X, \mathscr{A}, \mu)$. It is easy to show that every $\sigma$-finite measurable set is contained a.e. in the union of a countable subfamily of $\left\{X_{i}: i \in I\right\}$. From this observation it follows that for any $1 \leq p<$ $\infty$ and any Banach space $E, L^{p}(\mu, E) \simeq \sum_{i \in I} \oplus L^{p}\left(X_{i}, \mathscr{A}_{i}, \mu_{i}, E\right)$ (the direct sum of the spaces $\left.L^{p}\left(X_{i}, \mathscr{A}_{i}, \mu_{i}, E\right), i \in I\right)$, where $\mathscr{A}_{i}=\left\{A \cap X_{i}: A \in \mathscr{A}\right\}$, $\mu_{i}\left(A \cap X_{i}\right)=\mu\left(A \cap X_{i}\right), A \in \mathscr{A}, i \in I$.

Now, for each $i \in I$ let $\left(Y_{i}, \mathscr{B}_{i}, \hat{\mu}_{i}\right)$ be the perfect measure space associated with the finite measure space $\left(X_{i}, \mathscr{A}_{i}, \mu_{i}\right)$, and let $Y=\sum_{i \in I} \oplus Y_{i}$ be the topological direct sum of the topological spaces $Y_{i}, i \in I$. Clearly $Y$ is an extremally disconnected locally compact Hausdorff space, and the measure $\nu$, defined on the Borel algebra $\mathscr{B}$ of $Y$ by the equation $\nu(B)=\sum_{i} \hat{\mu}_{i}\left(B \cap Y_{i}\right)$, $B \in \mathscr{B}$, need not be regular but there exists a regular Borel measure on $Y$ which coincides with $\nu$ on each $\mathscr{B}_{i}, i \in I$.

Since each summand $Y_{i}$ is clopen in $Y$, for any subset $S$ of $Y$, int $S=$ $\bigcup_{i} \operatorname{int}\left(S \cap Y_{i}\right)$ and $\mathrm{Cl} S=\bigcup_{i} \mathrm{Cl}\left(S \cap Y_{i}\right)$. Thus, it follows that a subset is nowhere dense in $Y$ if and only if its intersection with each $Y_{i}$ is nowhere dense in $Y_{i}$, and since each $\hat{\mu}_{i}$ is a perfect measure on $Y_{i}$, we conclude that $\nu(B)=0$ for every nowhere dense Borel subset $B$ of $Y$. Clearly every nonempty clopen subset of $Y$ has strictly positive measure and contains another clopen set of finite measure. Hence $(Y, \mathscr{B}, \nu)$ is a perfect measure space and

$$
L^{p}(\mu, E) \simeq \sum_{i \in I} \oplus L^{p}\left(\hat{\mu}_{i}, E\right) \simeq L^{p}(\nu, E)
$$

for all $1 \leq p<\infty$, which completes the proof.

Observing that the measure space $(Y, \mathscr{B}, \nu)$ is decomposable we conclude the following corollary:

Corollary 1. If $E^{*}$ is separable then $L^{p}(\mu, E)^{*} \simeq L^{q}\left(\mu, E^{*}\right)$ for all $1<p<\infty$, $\frac{1}{p}+\frac{1}{q}=1$, and $L^{1}(\mu, E)^{*} \simeq L^{\infty}\left(\nu, E^{*}\right)$. In particular $L^{1}(\mu)^{*} \simeq L^{\infty}(\nu)$.

Corollary 2. Let $(X, \mathscr{A}, \mu)$ be an arbitrary measure space. Then there exists an extremally disconnected compact Hausdorff space $Z$ and a perfect Borel measure $\lambda$ on $Z$ such that for any Banach space $E$,

(i) $L^{p}(\mu, E) \simeq L^{p}(\lambda, E)$ for all $1 \leq p<\infty$,

(ii) $L^{1}(\mu, E)^{*} \simeq C\left(Z, E_{*}^{*}\right)$, and if $E^{*}$ is separable,

(iii) $L^{p}(\mu, E)^{*} \simeq L^{q}\left(\lambda, E^{*}\right)$ for all $1 \leq p<\infty, \frac{1}{p}+\frac{1}{q}=1$.

Consequently, $L^{\infty}\left(\lambda, E^{*}\right) \simeq C\left(Z, E_{*}^{*}\right)$.

Proof. Let $Z$ be the Stone-Čech compactification of the space $Y$ constructed in the proof of the theorem. Clearly $Z$ is extremally disconnected. Let $\nu$ be the perfect measure on $Y$ introduced earlier and define $\lambda$ on the Borel algebra $\mathscr{B}$ of $Z$ by the equation $\lambda(B)=\nu(B \cap Y), B \in \mathscr{B}$.

Since $Y$ is locally compact, it is open in $Z$, and thus, if $C$ is clopen in $Z$ then $C \cap Y$ is clopen in $Y$, and it is empty if and only if $C$ is empty. It now follows that $\lambda(C)>0$ for every nonempty clopen subset $C$ of $Z$, 
and every such set contains another clopen set with finite measure. If $B$ is a nowhere dense Borel subset of $Z$ then $B \cap Y$ is a nowhere dense Borel subset of $Y$ from which we conclude that $\lambda(B)=0$ for every nowhere dense Borel subset $B$ of $Z$. Hence $\lambda$ is a perfect Borel measure on $Z$ and obviously $L^{p}(\mu, E) \simeq L^{p}(\lambda, E)$ for all $1 \leq p<\infty$.

$(Z, \mathscr{B}, \lambda)$ is a decomposable measure space. Now the remaining assertions in the corollary follow from this fact and Cambern and Greim's theorem cited earlier.

Remark. The fact that for any Banach space $E, L^{1}(X, \mathscr{A}, \mu, E)^{*}$ can be realized as a space of continuous functions was first observed by Cambern and Greim [1, p. 375] for $\sigma$-finite measure spaces.

\section{REFERENCES}

1. M. Cambern and P. Greim, The dual of a space of vector measures, Math. Z. 180(1982), 373-378.

2. J. Diestel and J. J. Uhl, Jr., Vector measures, Math. Surveys Monogr. no. 15, Amer. Math. Soc., Providence, RI, 1977.

3. N. Dinculeanu, Vector measures, Pergamon Press, New York, 1967.

4. N. Dunford and J. J. Schwartz, Linear operators, Part I, Interscience, New York and London, 1967.

5. E. Hewitt and K. Stromberg, Real and abstract analysis, Springer-Verlag, New York, Heidelberg, and Berlin, 1965.

6. H. E. Lacey, The isometric theory of classical Banach spaces, Springer-Verlag, New York, Heidelberg, and Berlin, 1974.

7. J. Schwartz, A note on the space $L_{p}^{*}$, Proc. Amer. Math. Soc. 2(1951), 270-275.

Department of MAThematics, Yarmouk University, IRBid, Jordan 